\title{
Web-based Dynamic Delphi: a New Survey Instrument
}

\author{
JingTao Yao Wei-Ning Liu \\ Department of Computer Science \\ University of Regina \\ Regina, Saskatchewan \\ Canada S4S 0A2 \\ E-mail: [jtyao, liuwe200]@ cs.uregina.ca \\ URL: http://www.cs.uregina.ca/ jtyao
}

\begin{abstract}
We present a mathematical model for a dynamic Delphi survey method which takes advantages of Web technology. A comparative study on the performance of the conventional Delphi method and the dynamic Delphi instrument is conducted. It is suggested that a dynamic Delphi survey may form a consensus quickly. However, the result may not be robust due to the judgement leaking issues.
\end{abstract}

\section{INTRODUCTION}

The Delphi survey instrument is a way of collecting expert ideas and judgments through multi-round surveys. ${ }^{8,18}$ In each survey round, individual judgements are collected and summarized. It is hoped that those surveyed experts will evaluate and adjust their opinions upon the collective result available. It is also expected that a consensus could be reached at the end of a Delphi survey. A Delphi survey is mainly used in the situations where accurate information is unavailable or expensive to obtain, and human judgemental input is crucial. ${ }^{2,15,18,20,26}$

A Delphi survey uses mailed questionnaires with the feedback of individual contributions as its communication structure. ${ }^{6}$ The surveyed experts are totally isolated by an independent moderator who collects, filters and summarizes individual judgements. The feedback of each survey round is the only way that individual experts are informed of the opinions of the expert panel. It is believed that human social-emotional exchanges between individuals are helpful in facilitating consensus development and eliminating potential misunderstanding. ${ }^{16}$ However, it is difficult to implement such socialemotional interaction on the paper-and-pencil communication structure.

With the introduction of the Web technology, people are connected all over the world via the Internet. Online communication tools such as email, BBS, IRC, MUD, and Blog, provide more options and opportunities for human social-emotional interaction. In fact, many online survey tools are also available in the market. ${ }^{7,32}$ The ubiquity, real-time, low operating cost and convenience of Web features provide potential to release collective results dynamically between survey rounds. We thus propose a variation of the Delphi survey, namely the Web-based dynamic Delphi survey in this paper. Instead of providing feedbacks at the end of each survey round or iteration, collective opinions of panel experts will be released partially or fully dynamically between rounds. This paper also aims to study the features and effects of the newly proposed dynamic Delphi survey.

The organization of this paper is as follows. Section 2 reviews the Delphi survey. We will describe the process of the dynamic Delphi survey in Section 3. A comparative study of the conventional Delphi survey and the dynamic Delphi survey is also conducted. Section 4 develops the measures of the dynamic Delphi survey. In order to examine the effects of the dynamic Delphi survey, mathematical representations are presented in Section 5. Preliminary analysis results are presented in the same section. Finally, we conclude our findings in the last section.

\section{A REVIEW OF THE DELPHI SURVEY INSTRUMENT}

In this section, the procedure of the Delphi survey instrument is reviewed. We analyze the communication structure of the Delphi survey. Some disadvantages of the Delphi survey is also discussed.

A Delphi survey is usually completed through two or more survey rounds, without considering the problem-defining phase in which problems are formulated and the corresponding questionnaire is designed. ${ }^{20}$ As shown $\mathrm{n}$ Figure 1 , an action 


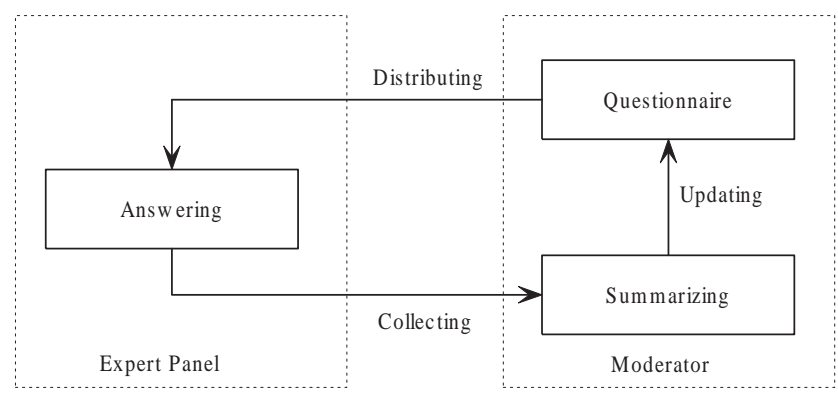

Figure 1. The procedure of a Delphi survey.

sequence of updating a questionnaire, distributing the questionnaire, answering the questionnaire, collecting individual answers, and then analyzing and summarizing results constitute a survey round. The collective result of each round is presented to the expert panel as a feedback. It is expected that the experts' answers will be influenced by their peers' opinions. Linstone and Turoff ${ }^{18}$ summarize the Delphi survey method in both technique and its objective:

Delphi may be characterized as a method for structuring a group communication process, so that the process is effective in allowing a group of individuals, as a whole, to deal with complex problems.

Row et al. ${ }^{27}$ characterizes the Delphi survey in a more detailed way:

It is a structured group process, in which individuals are required to give numerical judgments or forecasts over a number of rounds, with feedback being provided from the anonymous other members of the panel, and the final aggregate being taken as the process output.

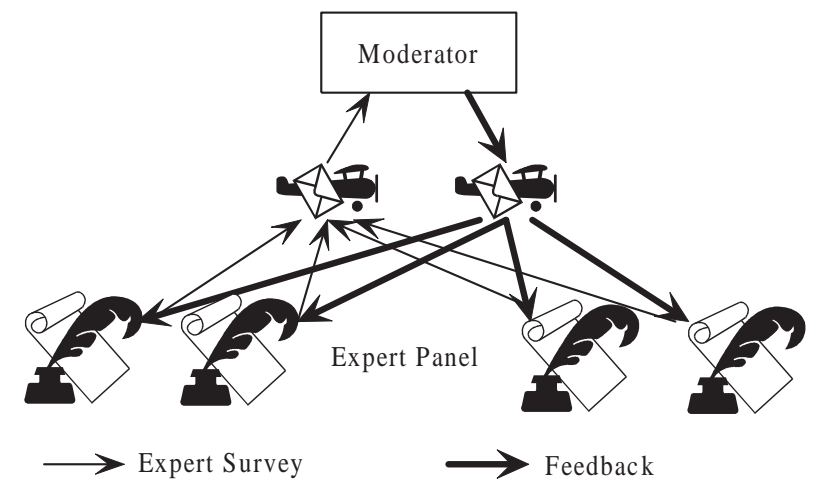

Figure 2. The communication structure of a Delphi survey.

There are two independent communication channels in a Delphi survey, the survey channel and the feedback channel, as shown in Figure 2. The survey channel and the feedback channel constitute a bridge between the panel experts. The experts' estimations first flow towards the moderator. The feedback from the moderator is then released only after all expert surveys have been completed in a round so that the experts can be informed of consistent collective information. Thus, the Delphi survey is characterized by a strict round or iteration structure.

However, the rigorous round structure of the Delphi survey may force all experts into a lockstep treatment of a problem. Only uniform feedback in each survey round may weaken the asynchrony of the Delphi which allows individuals with different cognitive abilities to parallel contribute to the different aspects of a complex problem. The experts' understanding may also be improved through analyzing sub-global views and considerations.

\section{THE DYNAMIC DELPHI SURVEY}

In this section, the dynamic Delphi survey is described according to the following two aspects, dynamic feedback releasing and 'direct' expert interaction. Dynamically releasing collective results between survey rounds with the dynamic Delphi 
survey may exert additional influence on individual experts.

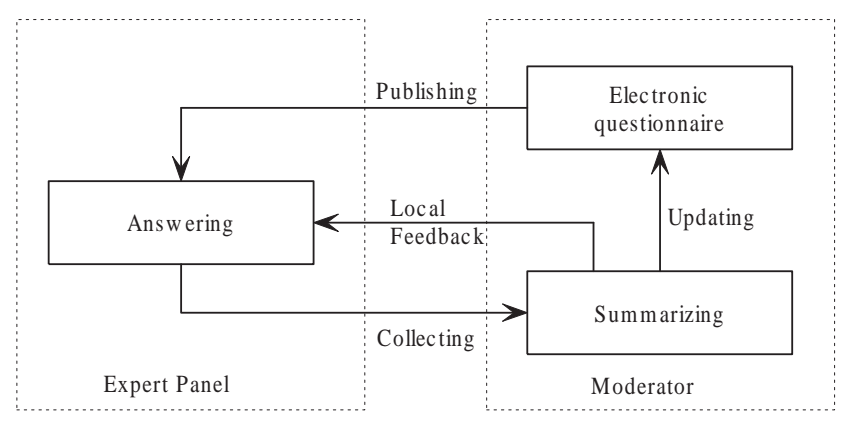

Figure 3. The procedure of a dynamic Delphi survey.

When an online survey is conducted, we may identify three ways of releasing collective results. The first way is the same as in the conventional Delphi survey: the moderator only releases the collective result after all questionnaires have been completed in a survey round. The above collective result is called as the global feedback and it should be accessible to all members in the expert panel. The second way is that when an individual contribution is submitted, the partial collective result may be updated and shown to the individual expert. The second way is similar to an online vote where any individual has an option to view the pulled opinion before or after his/her vote. However, in the case of a dynamic survey, an expert can only obtain the partial collective view after his/her opinion is submitted. Therefore, the preparation time for the next round survey would be longer. The third way of releasing collective result is that the partial collective result is revealed to all or some experts in the middle of a survey round. The partial collective result is called as a local feedback. Such a local view may impact individual experts' contributions.

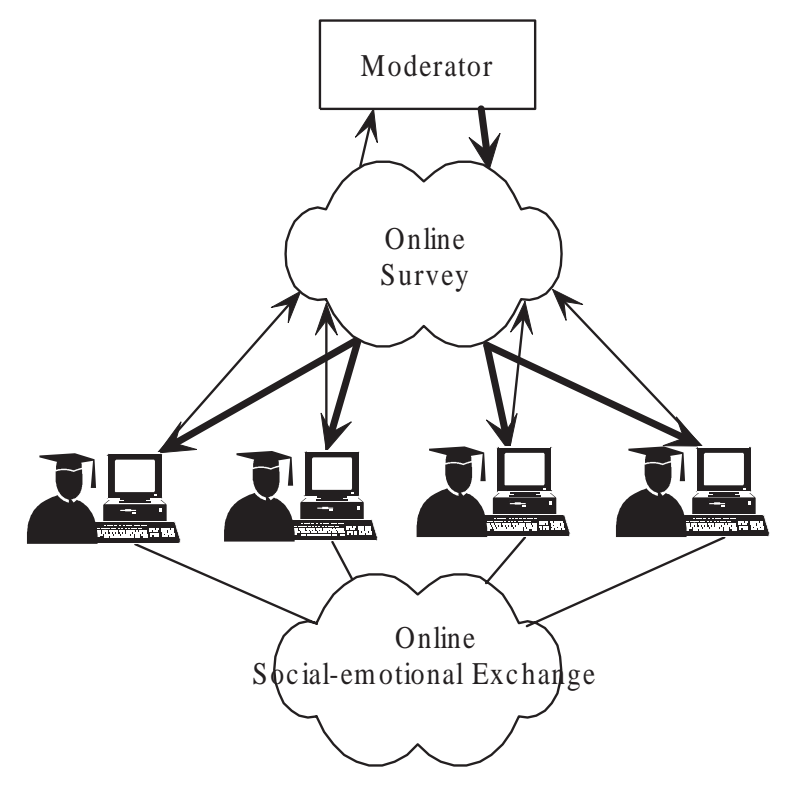

Figure 4. The communication structure of a dynamic Delphi survey.

With the dynamic Delphi survey instrument, it implies that some level of direct expert interactions may occur in some stages of during a survey. The establishment of direct expert interactions must be under the control of the moderator in order to prevent potential bias and undue social pressure. However, if all information exchanges between experts were through the moderator, they would be ineffective and difficult. An online communication environment can break through this dilemma by easily incorporating the use of pen names. ${ }^{17}$ A pen name can make a person be identified with a set of 
attributes without exposing his real status. These attributes may include: expertise, experience, character, viewpoint, and confidence which are hardly embedded into questionnaires of any form. By introducing direct expert interaction into a Delphi survey, there are seemingly intuitive advantages: the expert panel can obtain more understanding of why specific individuals are agreeing or disagreeing with certain concepts ${ }^{33}$; experts can be prompted to consider their positions more comprehensively, which may increase the opportunity of forming consensus.

As shown in Figure 3 and Figure 4, there are three independent communication channels in a dynamic Delphi survey: the survey channel, the feedback channel and the social-emotional exchange channel. The experts' judgements flow towards the moderator through the survey channel. The feedback from the moderator is released to all experts through the feedback channel at the end of each survey round. The local feedback may also be released to all or some experts through the feedback channel. The surveyed experts may directly communicate with each other under the control of the moderator. Although the dynamic Delphi survey has a round or iteration structure, such a round structure is not as strict as the conventional Delphi survey as collective opinions may be available between round.

On the other hand, although the moderator can decide when the direct interactions can be carried out and who can directly interact with each other, he/she cannot determine what the experts will talk about. One of a disadvantages is that some experts may intentionally or unintentionally expose their viewpoints to those who are still weighing their positions in a survey round. This phenomenon is called the individual judgement leaking. The judgemental leaking may impact the decision-making of some experts, and may alter the results of a dynamic Delphi survey.

\section{THE MEASURES OF THE DYNAMIC DELPHI SURVEY}

There are some empirical knowledge on the consensus formation in a dynamic Delphi survey. For example, Salancik 28 reported that bad Delphi questionnaires may yield low consensus; Rowe ${ }^{27}$ investigated the complex relations of individual opinion change and judgemental accuracy of the Delphi survey in forecasting. In this section, we study the consensus formation or the response stability in the dynamic Delphi survey.

\subsection{Individual confidence}

Individual opinion changes are measurable in terms of personal willingness to alter their judgements over Delphi rounds. Without considering the panelist's feelings towards questionnaires, the willingness is referred to indvidual's belief in their own expertise and in their judgements. The belief in personal expertise can be assumed to be invariable during a Delphi. The belief in personal judgement may change when individuals use the opportunity afforded by each Delphi round to reflect on their own previous judgments or to take the group feedback into account. Although the belief in personal judgement is unnecessarily related to the belief in personal expertise, we still see them as an independent variable, for their individual effects are hardly discerned. We call the variable as the individual confidence. According to Rowe, ${ }^{25}$ it can be predicted that high initial confidence will support low propensity to make judgement change over Delphi rounds. The individual belief changes will prompt individuals to change their judgements in subsequent rounds.

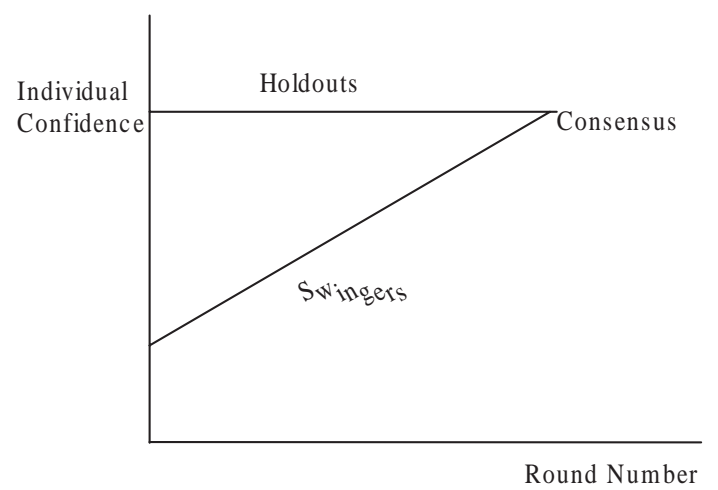

Figure 5. A quantitative illustration of individual confidence changes of the swingers according to the theory of errors. 
In principle, the individual confidence changes can be explained the theory of errors. ${ }^{11}$ The theory of errors is seen as an axiomatic explanation of the Delphi technique. ${ }^{25}$ By Parenté ${ }^{22}$ the Delphi panel can be divided into two sets: the holdout set and the swinger set. The former are assumed to comprise the more-knowledgeable panelists, and the latter the less-knowledgeable ones. In the face of the group feedback, the swingers will be drawn towards the group view, whereas the holdouts will be confident of their expertise and maintain their judgements. When the dogmatic and self-opinionated cases concerned by Mulgrave ${ }^{21}$ are not involved, we can assume that the holdouts have higher and steady individual confidences, and their judgements are reliable and will not change over rounds. The swingers are assumed to have lower and unsteady individual confidences, and their judgements are unreliable to some extent. In order to increase the individual confidence, the swingers are inclined to change their opinions towards the group view. When the individual confidences of the swingers match or are closed to the ones of the handouts, a consensus or a steady opinion distribution is deemed to be achieved. The result of their efforts is that the judgemental reliability of the swingers will improve over Delphi rounds and the group view will subsequently improve.

We also assume that the individual confidence of panelists derives from the self-assessment on their expertise and judgements, and the external information from which the swingers can realize their relative lack of knowledge. Parenté ${ }^{23}$ illustrated that the self-assessment can alone result in improved accuracy of forecasting, but no objective factors can guarantee the accuracy of self-assessment. In a conventional Delphi, the information towards the panelists is controlled by the moderator, which is not always comprehensive in each case. By establishing direct member interaction channels for socializing among the panel, the dynamic Delphi provides extra opportunity for the swingers to calibrate their individual confidence. Therefore, we can speculate that the dynamic Delphi may be much more in favor of improving the appropriateness of individual judgement change than the conventional Delphi.

\subsection{Feedback}

Rowe $^{27}$ argued that the Delphi can improve judgemental reliability because of the group feedback provided between rounds. Rowe ${ }^{25}$ identified a type of group feedbacks which have more profound reasons for the group view, and found that in the face of the reasons feedback, individual judgement changes tend to be for the better, leading to a reduction in error. ${ }^{27}$ On the other hand, the dynamic Delphi can provide more comprehensive information feedbacks for the panelists by allowing the direct member interactions. However, the uncontrollability of contents of the direct interaction may lead to more complex outcomes.

If the direct member interaction does not result in the judgemental short-circuit, and is merely ordinary social-emotional exchange, what the direct interaction transfers can be seen as a reasons supplement of the group feedback. In this case, we can assume that panelists face roughly the same average degree of opinion pull against their positions. In the conventional Delphi, the moderator has to alone bear the load of preparing the reasons feedback while in the dynamic Delphi, part of the workload can be distributed over the whole panel. Hence, the development of reasons feedbacks in the conventional Delphi obviously costs higher than in the dynamic Delphi.

If the direct member interaction brings the judgemental short-circuit, some uncertainties may be introduced because the panelists face different degrees of opinion pull against their position in each round. Now we consider the judgemental short-circuit procedure. In each round of this group process, those panelists who earlier submit their contributions can access less local feedbacks before making their decisions than other panelists. On the contrary, the later the panelists submit their contributions, the more local feedbacks they can receive before making their decisions. In order to clarify the potential issue brought by the "order" of submitting individual contributions, we make the following assumptions: in each round,

- the holdouts will not change their judgements;

- the swingers will not change their judgements unless a holdout has published his judgement before making their decisions.

We then give an example to illustrate that the accuracy of the judgemental short-circuit procedure relies on the the order of submitting individual contributions in each round. Assume that there are five individuals participating in the judgemental short-circuit procedure where one panelist is a holdout and the rest are swingers. We also label the judgement of the holdout as "correct" and label the initial judgements of the swingers as "error". In addition, the group process is assumed to enter the second round. 
- If the holdout is the first one submitting judgement, all swingers will change their initial judgements towards the judgement of the holdout. Correspondingly, the group view will close to the correct judgement.

- If the holdout is the third one submitting judgement, half swingers will not change their error judgements. Consequently, the group view will not be better than the first case.

- If the holdout is the last one submitting judgement, all swingers will not change their error judgements. Obviously, the group view will be the worst among the three cases.

In this section, we analyze the consensus formation and reliability of the dynamic Delphi survey in terms of the theory of errors. Two variables: individual confidence and feedback are studied in the context of a dynamic Delphi survey. The individual confidence is the drive that impels individual judgement changes. The feedback reflects the strength of external factors in individual judgement changes. Based on these two variables, the potential advantages and disadvantages of the dynamic Delphi survey in comparison with the conventional Delphi are discussed. In addition, we have to point out that the conclusions obtained here are only some speculations about the dynamic Delphi survey based on the theory of errors and the empirical conclusions of the conventional Delphi. They still need to be verified in practice.

\section{A QUANTITATIVE ANALYSIS OF THE DYNAMIC DELPHI SURVEY}

In this section, we will investigate the cost of carrying out a dynamic Delphi survey. Most of academic explorations of the Delphi drew their findings from empirical examinations or application instances. ${ }^{26}$ For instance, Linstone and Turoff ${ }^{18,33}$ studied the additional opportunities added by the introduction of Computer Mediated Communication Systems (CMC) into the Delphi. Both of them paid attention to the economic advantages of computerized conferencing over the conventional Delphi. Synder ${ }^{31}$ compared the Internet and mail approaches in terms of time and expenditure. Only Dalkey ${ }^{10}$ strived to develop some mathematically rigorous underpinnings for the Delphi.

There are different explanations and measurements of the term of cost, and each of them is suitable for distinct contexts. In our study, "round number" is adopted to measure the cost of the dynamic Delphi. Linstone ${ }^{18}$ reported that in many cases, three rounds are sufficient to attain stability of responses; further rounds tend to show very little change and excessive repetition is unacceptable to participants. Obviously, cost is related to the mental endurance of individuals facing repetitive inquiry. Another issue challenging us is that there is not an explicit correspondence between round number and a Delphi procedure. Our solution is to establish a mathematical model to stimulate individual judgement changes and the formation of group view.

Dalkey ${ }^{10}$ suggested that a process be involved in each round of a Delphi, and it can be expressed as

$$
G=G[I, E, R]
$$

where $I$ represents the panel; $E$ the problem space addressed by the Delphi; $R$ the response space; $G$ the group view. Square brackets are used to emphasize the fact that the formulation of group view cannot be expressed as a simple functional relationship. Our model is an instantiation of (1), which simulates the individual judgement changes and the group view formulation in the judgemental leaking procedure described in Section 3. We first make the following assumptions:

- $E=[0,1]$, and a judgement on $E$ is a selection from $E$.

- $I=\left\{I_{i} \mid 1 \leq i \leq N\right\}$ has $N$ panelists.

- $M(M \geq 1)$ the round numbers.

- $R=\left\{r_{i, j} \mid 1 \leq i \leq N, 1 \leq j \leq M\right\}$, where $r_{i, j}$ is the judgement of panelist $I_{i}$ in $j^{\text {th }}$ round. In each round, panelists will in turn submit their judgements in an order from 1 to $N$.

- $k_{i, j} \in[0,1]$ the individual confidence coefficient of panelist $I_{i}$ in $j^{\text {th }}$ round. The less $k_{i, j}$, the higher the individual confidence of $I_{i}$ in $j^{t h}$ round. $k_{i, 1}=0$ indicates that $I_{i}$ is a holdout who will not change his judgement over rounds. $k_{i, 1}>0$ indicates that $I_{i}$ is a swinger who will change his judgement in subsequent rounds. We further assume that $k_{i, j}$ is a descending function of $j$, i.e. the swingers will increase their individual confidence over rounds. 
- $G_{j}$ the group view developed by the moderator in $j^{\text {th }}$ round.

- $g_{i, j}$ the local view formed by panelist $I_{i}$ in $j^{\text {th }}$ round.

The process involved in the $j^{\text {th }}$ round of the judgemental short-circuit procedure can be represented by a set of functional relations:

\section{Individual judgement}

$$
r_{i, j}=\Upsilon\left(r_{i, j-1}, G_{j-1}, g_{i, j}, k_{i, j-1}\right),
$$

where $\Upsilon$ represents the process of individual opinion change. We further assume that $k_{i, j-1}$ impels the change from $r_{i, j-1}$ to $r_{i, j}$, whereas the variational content is determined by $r_{i, j-1}, G_{j-1}$ and $g_{i, j}$.

\section{Group view}

$$
G_{j}=\Phi\left(r_{1, j}, \ldots, r_{n, j}\right),
$$

where $\Phi$ represents the process of extracting the group view from individual judgements.

\section{Local view}

$$
g_{i, j}=\phi\left(r_{1, j}, \ldots, r_{i-1, j}\right),
$$

where $\phi$ represents the process in which $I_{i}$ develops his own local view in $j^{\text {th }}$ round.

In fact, the model is still suitable for stimulating the conventional Delphi survey described in Section 2 if we exclude the local view $g_{i, j}$ from the above formulae. In order to use our model to stimulate the judgemental short-circuit procedure and the conventional Delphi procedure, we need to further instantiate the model.

We first assume that the individual confidence will change according to the following rule in both procedures:

$$
k_{i, j}=k_{i, j-1}-\frac{1}{2^{j-1}}, \quad(j>1)
$$

Particularly, $\kappa_{i, j-1}<\frac{1}{2^{j-1}}$ means that $\kappa_{i, j}=0$.

The individual judgement change is estimated by

$$
\begin{aligned}
& r_{i, j}=r_{i, j-1}+k_{i, j} \times\left(G_{j}-r_{i, j-1}\right), \text { or } \\
& r_{i, j}=r_{i, j-1}+k_{i, j} \times\left[\left(G_{j}-r_{i, j-1}\right)+\left(g_{i, j}-r_{i, j-1}\right)\right] .
\end{aligned}
$$

\subsection{Scenario I: the conventional Delphi process}

\section{Round 1}

$$
G_{1}=\sum_{i=1}^{N} \frac{r_{i, 1}}{N} .
$$

\section{Round 2}

$$
\begin{aligned}
k_{i, 2} & =k_{i, 1}-\frac{1}{2} . \\
r_{i, 2} & =r_{i, 1}+k_{i, 2} \times\left(G_{1}-r_{i, 1}\right) . \\
G_{2} & =\left(1+\sum_{i=1}^{N} \frac{k_{i, 2}}{N}\right) \times G_{1}-\sum_{i=1}^{N} \frac{\left(k_{i, 2} \times r_{i, 1}\right)}{N} .
\end{aligned}
$$




\section{Round 3}

$$
\begin{aligned}
k_{i, 3} & =k_{i, 2}-\frac{1}{4} . \\
r_{i, 3} & =r_{i, 2}+k_{i, 3} \times\left(G_{2}-r_{i, 2}\right) \\
& =\left(1-k_{i, 3}\right) \times r_{i, 2}+k_{i, 3} \times G_{2} \\
G_{3} & =\left(1+\sum_{i=1}^{N} \frac{k_{i, 3}}{N}\right) \times G_{2}-\sum_{i=1}^{N} \frac{\left(k_{i, 3} \times r_{i, 2}\right)}{N} .
\end{aligned}
$$

\section{Round j}

$$
\begin{aligned}
k_{i, j} & =k_{i, 1}-1+\frac{1}{2^{j}} . \\
r_{i, j}-r_{i, j-1} & =\left(k_{i, 1}-1+\frac{1}{2^{j}}\right) \times\left(G_{j-1}-r_{i, j-1}\right) . \\
G_{j}-G_{j-1} & =\sum_{i=1}^{N} \frac{\left(k_{i, 1}-1+\frac{1}{2^{j}}\right) \times\left(G_{j-1}-r_{i, j-1}\right)}{N} .
\end{aligned}
$$

\subsection{Scenario II: the judgemental short-circuit procedure}

Here, we use $r_{i, 1}$ represents the initial opinion of panelist $I_{i}$ and use $s_{i, j}$ represents the opinion change of $I_{i}$. In addition, $S_{j}$ is used to represent the group view of $j^{\text {th }}$ round.

\section{Round 1}

$$
\begin{aligned}
s_{1,1} & =r_{1,1}, \\
g_{1,1} & =r_{1,1}, \\
s_{i, 1} & =r_{i, 1}+k_{i, 1} \times\left(g_{i-1,1}-r_{i, 1}\right), \quad(i \geq 2) \\
g_{i, 1} & =\sum_{t=1}^{i} \frac{s_{t, 1}}{i} . \\
S_{1} & =\sum_{i=1}^{N} \frac{s_{i, 1}}{N}, \\
& =\sum_{i=1}^{N} \frac{r_{i, 1}}{N}+\sum_{i=2}^{N} \frac{k_{i, 1}}{N} \times\left(g_{i-1,1}-r_{i, 1}\right), \\
& =G_{1}+\sum_{i=2}^{N} \frac{k_{i, 1}}{N} \times\left(g_{i-1,1}-r_{i, 1}\right) .
\end{aligned}
$$

We now compare $s_{i, 1}(10)$ and $r_{i, 2}(8)$ :

$$
s_{i, 1}-r_{i, 2}=k_{i, 1} \times\left(g_{i-1,1}-r_{i, 1}\right)-\left(k_{i, 1}-\frac{1}{2}\right) \times\left(G_{1}-r_{i, 1}\right) .
$$

$g_{i-1,1}$ is indirectly related to $r_{i, 1}$, and $G_{1}$ is the arithmetic average of $r_{i, 1}$ which implies a statistical conclusion: $s_{i, 1}>r_{i, 2}$. It means that the change degrees of individual opinions in round one of the judgemental leaking procedure are usually bigger than the ones of individual opinions from round one to round two in the conventional Delphi. Similarly,

$$
S_{1}-G_{2}=\sum_{i=2}^{N} \frac{k_{i, 1}}{N} \times\left(g_{i-1,1}-r_{i, 1}\right)-\sum_{i=1}^{N} \frac{k_{i, 2}}{N} \times\left(G_{1}-r_{i, 1}\right),
$$

which implies a statistical conclusion: $S_{1}>G_{2}$. 


\section{Round 2}

$$
\begin{aligned}
s_{1,2} & =s_{1,1}+k_{1,2} \times\left(S_{1}-s_{1,1}\right) \\
g_{1,2} & =s_{1,2} \\
s_{i, 2} & =s_{i, 1}+k_{i, 2} \times\left[\left(S_{1}-s_{i, 1}\right)+\left(g_{i-1,2}-s_{i, 1}\right)\right], \quad(i \geq 2) \\
g_{i, 2} & =\sum_{t=1}^{i} \frac{s_{t, 2}}{i} . \\
S_{2} & =\sum_{i=1}^{N} \frac{s_{i, 2}}{N} \\
& =\sum_{i=1}^{N} \frac{s_{i, 1}}{N}+\sum_{i=1}^{N} \frac{k_{i, 2} \times\left(S_{1}-s_{i, 1}\right)}{N}+\sum_{i=1}^{N} \frac{k_{i, 2} \times\left(g_{i-1,2}-s_{i, 1}\right)}{N} . \\
S_{2}-S_{1} & =\sum_{i=1}^{N} \frac{k_{i, 2} \times\left(S_{1}-s_{i, 1}\right)}{N}+\sum_{i=1}^{N} \frac{k_{i, 2} \times\left(g_{i-1,2}-s_{i, 1}\right)}{N} .
\end{aligned}
$$

By comparing $S_{2}-S_{1}(12)$ and $G_{2}-G_{1}(9)$, a statistical conclusion of $\left(S_{2}-S_{1}\right)>\left(G_{2}-G_{1}\right)$ likely exists for $s_{i, 1}$ is indirectly related to $g_{i-1,2}$.

\section{Round 3}

$$
\begin{aligned}
s_{i, 3} & =s_{i, 2}+k_{i, 3} \times\left[\left(S_{2}-s_{i, 2}\right)+\left(g_{i-1,3}-s_{i, 2}\right)\right], \quad(i \geq 2) \\
g_{i, 3} & =\sum_{t=1}^{i} \frac{s_{t, 3}}{i} . \\
S_{3} & =\sum_{i=1}^{N} \frac{s_{i, 3}}{N}, \\
& =\sum_{i=1}^{N} \frac{s_{i, 2}}{N}+\sum_{i=1}^{N} \frac{k_{i, 3} \times\left(S_{2}-s_{i, 2}\right)}{N}+\sum_{i=1}^{N} \frac{k_{i, 3} \times\left(g_{i-1,3}-s_{i, 2}\right)}{N} . \\
S_{3}-S_{2} & =\sum_{i=1}^{N} \frac{k_{i, 3} \times\left(S_{2}-s_{i, 2}\right)}{N}+\sum_{i=1}^{N} \frac{k_{i, 3} \times\left(g_{i-1,3}-s_{i, 2}\right)}{N} .
\end{aligned}
$$

By comparing $S_{2}-S_{1}(14)$ and $G_{2}-G_{1}(9)$, the statistical conclusion of $\left(S_{3}-S_{2}\right)>\left(G_{3}-G_{2}\right)$ still likely exists.

In this section, we comparatively study a dynamic Delphi survey and a conventional Delphi survey. The results imply that the introduction of the direct expert interaction may damage the performance of the Delphi when experts are impacted by different local views. Local views produce uneven opinion pull in the panel which results in sharp change of individual opinions and bad convergence of the group views.

\section{CONCLUSIONS}

In this paper, we propose a variation of the Delphi survey technique, namely, the dynamic Delphi survey instrument. This variation is derived from the observation that that social-emotional exchanges among panelists may be of help in facilitating consensus development and eliminating potential misunderstanding. The panelists are allowed to obtain collective opinions dynamically, i.e., between each survey round.

We conducted a comparatively study on procedures and characteristics of the conventional Delphi and the dynamic Delphi method. A set of functional relations are established to describe the process involved in each round of a Delphi survey. It is suggested that a dynamic Delphi survey may form a consensus quickly. However, a dynamic Delphi survey may result in sharp changes of individual opinions and worse convergence of the collective group view when panelists are impacted by different local views. The reason may be that local views produce uneven opinion pull in the panel. 


\section{REFERENCES}

1. Ayton, P., Ferrell, W. R. and Stewart, T. R. Commentaries on the Delphi technique as a forecasting tool: issues and analysis by Rowe and Wright, International Journal of Forecasting, 15, pp.377-381, 1999.

2. Best, R. J. An experiment in Delphi estimation in marketing decision-making, Journal Marketing Research, 11, pp.448-452, 1974.

3. Boje, M. E. and Murnighan, J. K. Group confidence pressures in iterative decisions, Management Science, 28(10), pp.1187-1196, 1982.

4. Bonner, B. L., Baumann, M. R. and Dalal, R. S. The effects of member expertise on group decision-making and performance, Organizational Behavior and Human Decision Processes, 88(2), pp.719-736, 2002.

5. Brockhoff, $\mathrm{K}$. The performance of forecasting groups in computer dialogue and face-to-face discussion in: Linstone and Turoff (Eds.) The Delphi method: techniques and applications, Massachusetts Reading: Addison-Wesley, 1975.

6. Cho, H. K., Turoff, M. and Hiltz, S. R. The impacts of Delphi communication structure on small and medium sized asynchronous groups: preliminary results, Proceedings of the 36th Hawaii International Conference on System Sciences (HICSS'03), 2002.

7. Chou, C. Developing the e-Delphi System: a Web-based forecasting tool for educational research, British Journal of Educational Technology, 33(2), pp.233-236, 2002.

8. Dalkey, N. C. and Helmer, O. An experimental application of the Delphi method to the use of experts. Management Science, 9, pp.458-467, 1963.

9. Dalkey, N. C. An experimental study of group opinion, Rand RM-5888-PR, Rand Corporation, 1969.

10. Dalkey, N. C., Brown, B. and Cochran, S. Use of self-ratings to improve group estimates, Technological Forecasting, 1(3), pp.91-283, 1970.

11. Dalkey, N. C. Toward a theory of group estimation in: Linstone and Turoff (Eds.) The Delphi method: techniques and applications, Massachusetts Reading: Addison-Wesley, 1975.

12. Deshpande, A. M., Shiffman, R. N. and Nadkarni, P. M. Metadata-driven Delphi rating on the Internet, Computer Mmethods and Programs in Biomedicine, 77(1), pp.49-56, 2005.

13. Fink, A. How to analyze survey data, Sage Publications, 1995.

14. Gordon, T. J. and Helmer, O. Report on a long range forecasting study, Rand Paper P-2982, Rand Corporation, 1964.

15. Grabbe, E. M. and Pyke, D. L. An evaluation of the forecasting of information processing technology and applications, Technological Forecasting and Social Change , 4(2), pp.143, 1972.

16. Hiltz, S. R., Johnson, K. and Turoff, M. Experiments in group decision making: communication process and outcome in face-to-face vs. computerized conferences, Human Communication Research, 13(2), pp.225-253, 1986.

17. Hiltz, S. R., Turoff, M. and Johnson, K. Experiments in group decision making, 3: Disinhibition, Deindividuation, and Group Process in Pen Name and Real Name Computer Conferences, Journal of Decision Support Systems, 5, pp.217-232, 1989.

18. Linstone, H. A. and Turoff, M. (Eds.) The Delphi method: techniques and applications, Massachusetts Reading: Addison-Wesley, 1975.

19. Martino, J. P. The precision of Delphi estimates, Technological Forecasting, 1(3), pp.99-293, 1970.

20. Martino, J. P. Technological forecasting for decision making, Elsevier Science Publishing, New York, 1983.

21. Mulgrave, N. W. and Ducanis, A. J. Propensity to change responses in a Delphi round as a function of dogmatism in: Linstone and Turoff (Eds.) The Delphi method: techniques and applications, Massachusetts Reading: AddisonWesley, 1975.

22. Parenté, F. J., Anderson, J. K. Delphi inquiry systems in: Wright and Ayton (Eds.) Judgmental Forecasting, Wiley, Chichester, pp.129-156, 1987.

23. Parenté, F. J., Anderson, J. K., Myers, P. and O'Brien, T. An examination of factors contributing to Delphi accuracy, Journal of Forecasting, 3(2), pp.173-182, 1984.

24. Prietula, M. J., Feltovich, P. J. and Marchak, F. Factors influencing analysis of complex cognitive tasks: a framework and example from industrial process control, Human Factors, 42(1), pp.56-74, 2000.

25. Rowe, G. and Wright, G. The impact of task characteristics on the performance of structured group forecasting techniques, International Journal of Forecasting 12, pp.73-90, 1996.

26. Rowe, G. and Wright, G. The Delphi technique as a forecasting tool: issues and analysis, International Journal of Forecasting, 15, pp.353-375, 1999. 
27. Rowe, G., Wright, G. and McColl, A. Judgment change during Delphi-like procedures: the role of majority influence, expertise, and confidence, Technological Forecasting \& Social Change, 72, pp.377-399, 2005.

28. Salancik, J. R., Wenger, W. and Helfer, E. The construction of Delphi event statements, Technological Forecasting and Social Change, 3(1), pp.65-73, 1971.

29. Salancik, J. R. Assimilation of aggregated inputs into Delphi forecasts: a regression analysis, Technological Forecasting and Social Change, 5(3), pp.48-243, 1973.

30. Scheibe, M., Skutsch, M. and Schofer, J. Experiments in Delphi methodology in: Linstone, H. A. and Turoff, M.(Eds.), The Delphi method: techniques and applications, Addison-Wesley, MA Reading, 262-287, 1975.

31. Snyder-Halpern, R., Thompson, C. B. and Schaffer, J. Comparison of mailed vs. Internet applications of the Delphi technique in clinical informatics research, Journal of the American Medical Informatics Association, Suppl. S, pp.809$813,2000$.

32. Taylor, H. Does internet research work? comparing online survey results with telephone survey, International Journal of Market Resarch 42(1), pp.51-63, 2000.

33. Turoff, M. and Hiltz, S. R. Computer-based Delphi processes, http://eies.njit.edu/ turoff/Papers/delphi3.html (accessed 2005).

34. Wolfgang, W. Delphi-methode, Gestaltung und Potential für betriebliche Prognoseprozesse, Schriftenreihe Wirtschaftswissenschaftliche Forschung und Entwicklung, München, 1978.

35. Wood, A. F. and Smith, M. J. Online communication: linking technology, identity, and culture, Lawrence Erlbaum Associates, Inc., Mahwah, New Jersey, 2005. 